\section{Die Rhombencephalosynapsis als seltene Anomalie in der hinteren Schädelgrube}

Die Rhombencephalosynapsis ist eine seltene Kleinhirnfehlbildung, welche durch eine Fusion der zerebellären Hemisphären und das Fehlen bzw. eine Hypoplasie des Kleinhirnwurms gekennzeichnet ist. Assoziierte supratentorielle Fehlbildungen bestehen in einem unterschiedlichen Ausmaß und korrelieren mit der Schwere der klinischen Behinderung (Barkovich A. J. Pediatric Neuroimaging. $3^{\text {rd }}$ ed. New York, Raven Press 2000: $346 \mathrm{f})$.

\section{Fallbericht}

Ein 5-jähriges Mädchen wurde wegen einer unklaren Bewusstlosigkeit und klinischen Hirndruckzeichen stationär aufgenommen. Anamnestisch hatte es unmittelbar postnatal einen V-P-Shunt bei Verschlusshydrozephalus erhalten. Eine weiterführende bildgebende Diagnostik erfolgte bisher nicht. Klinisch bestanden akute Hirndruckzeichen. Außerdem waren eine mentale und motorische Retardierung bekannt. Im kranialen Notfall-CT zeigte sich ein Hydrozephalus bei Shuntinsuffizienz im Rahmen einer komplexen Hirnfehlbildung. Es erfolgte eine sofortige ShuntRevision. Zwischenzeitlich wurde das Kind reanimationspflichtig. Postoperativ erfolgte ein MRT zur Abklärung einer intrazerebralen Hypoxiefolge sowie zur Einordnung der zerebralen Fehlbildung. Das kraniale MRT am $1.5 \mathrm{~T}$ Siemens Vision zeigte ein Fehlen des Vermis mit Verschmelzung der zerebellären Hemisphären sowie der medialen Kleinhirnschenkel bei Hypoplasie der hinteren Schädelgrube. Der 4. Ventrikel wies eine Tränenform auf (Abb.1). Weiterhin fand sich eine komplexe Mittellinienfehlbildung mit Aplasie des Septum pellucidum und der Fornices und konsekutiver Verschmelzung des hypoplastischen Seitenventrikelsystems (Abb. 2). Es fielen eine partielle Fusion der Nuclei caudati und der Thalami (Abb. 2) sowie der Colliculi sup. et inf. auf. Das dorsale Drittel des Corpus callosum inclusive Splenium war aplastisch (Abb.3). Der Aquaeductus Sylvii verblieb ohne Flussnachweis. Die Kleinhirntonsillen standen tief im Foramen magnum (Abb.3). Außerdem zeigten sich eine kortikale Dysplasie mit komplexen Gyrierungsstörungen (Poly-

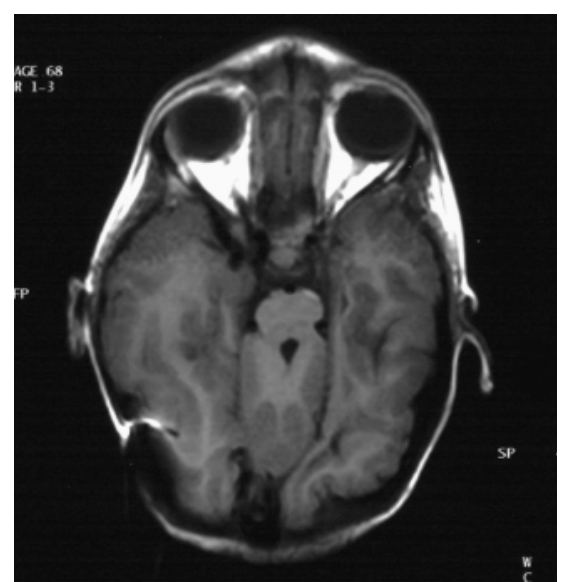

Abb. 1 Transversale T1-SE-Sequenz (TR $630 \mathrm{~ms}$; TE $14 \mathrm{~ms}$ ). Tränenform des 4. Ventrikels durch Verschmelzung der medialen Pedunculi cerebellares sowie der Nuclei dentati und der Kleinhirnhemisphären bei Fehlen des Vermis cerebelli. Asymmetrische Verschmächtigung des temporalen Marklagers mit Polymikrogyrie. Liegendes Shuntsystem rechts retroaurikulär mit Suszeptibilitätsartefakten.

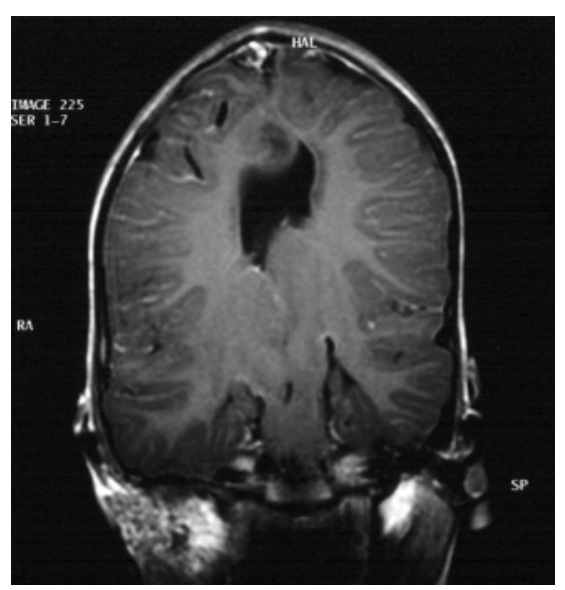

Abb. 2 Koronare T1-SE-Sequenz (mit KM) (TR $462 \mathrm{~ms}$; TE $12 \mathrm{~ms}$ ). Aplasie des Septum pellucidum mit Verschmelzung der hypoplastischen Seitenventrikel. Asymmetrie und partielle Verschmelzung der Thalami bei nicht abgrenzbarem 3. Ventrikel. Verschmälerung des periventrikulären Marklagers. Polymikrogyrie.

mikrogyrie) und assoziierten prominenten kortikalen Gefäßen sowie eine Falxdysplasie. In der echoplanaren Bildgebung fiel eine Diffusionsstörung in den Basalganglien und im Kortex beidseits auf (Abb.4), welche als Zeichen einer

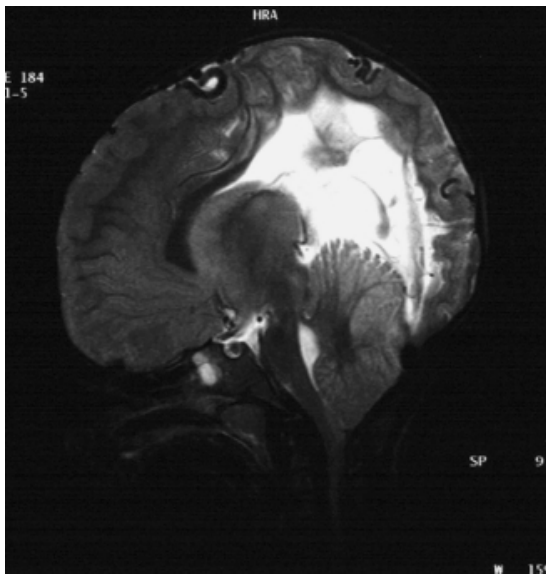

Abb. 3 Sagittale T2-TCSE-Sequenz mit Fettsättigung (TR $7400 \mathrm{~ms}$; TE $115 \mathrm{~ms} ; \alpha 160^{\circ}$ ). Aplasie des dorsalen Anteils des Corpus callosum. Fehlender Flussnachweis im Aquädukt. Tief im Foramen magnum stehende Kleinhirntonsillen. Deutlich erweiterte, geschlängelt verlaufende kortikale Gefäße.

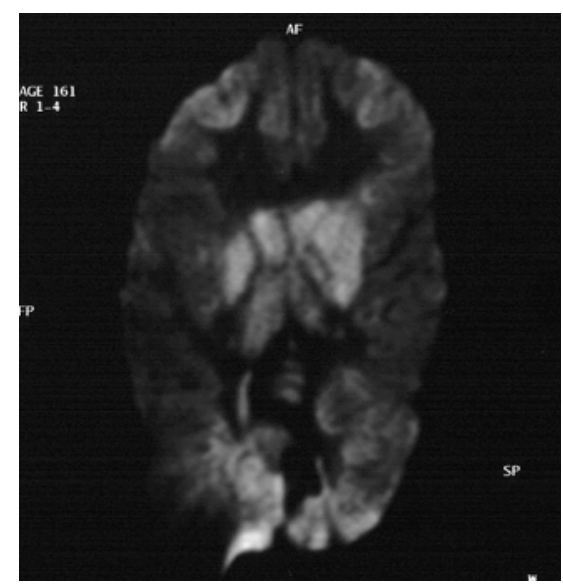

Abb.4 Transversale EPI-Sequenz (TR 3900 ms; TE 100/5 ms; diffusionsgewichtet). Diffusionsstörung in den Stammganglien bds. sowie im bifrontalen und bioccipitalen Kortex als Zeichen der diffusen Hypoxie. Asymmetrie der Nuclei caudati, Nuclei lentiformes und Thalami bds. Suszeptibilitätsartefakt durch VP-Shunt retroaurikulär rechts.

diffusen Hypoxie gewertet wurde. Das Kind verstarb 2 Tage nach der MR-Untersuchung. Eine Obduktion wurde nicht durchgeführt.

\section{Diskussion}

Die Rhombencephalosynapsis ist eine sehr seltene komplexe Malformation in der hinteren Schädelgrube. In der Literatur sind bisher 39 Fälle beschrieben (Montull C et al., Neuroradiology 2000; 42: 52; Sener R. N. Comput Med Imaging 
Graph 2000; 24 (4): 277). Die Rhombencephalosynapsis ist charakterisiert durch eine Apposition bzw. Fusion der Nuclei dentati und eine Fusion der Kleinhirnhemisphären. Durch Agenesie bzw. Hypogenesie des Wurmes und das Fehlen des Nodulus entsteht eine Tränenform des 4 . Ventrikels. Weiterhin werden in variabler Ausprägung eine Fusion der Pedunculi cerebellares mediales sowie der Colliculi sup. et inf., Heterotopien der grauen Substanz, eine Stenose oder Atresie des Aquaeductus cerebri und eine Hypo- oder Aplasie der Nuclei olivares inferiores beschrieben (Barkovich A. J. Pediatric Neuroimaging. $3^{\text {rd }}$ ed. New York, Raven Press 2000: 346f; Montull C et al., Neuroradiology 2000; 42: 52).

Diese Kleinhirnanomalien zeigen sich bei allen bisher beschriebenen Fällen weitgehend konstant. Dagegen sind die assoziierten Anomalien der supratentoriellen Mittellinienstrukturen vielfältig. Es handelt sich dabei insbesondere um Malformationen der ventralen Induktion, da diese zeitgleich mit der Entwicklung des Kleinhirns auftritt (Truwit CL. et al. Am J Neuroradiol 1991; 12: 957). In der Literatur werden ein Fehlen bzw. eine Hypoplasie der vorderen Kommissur und des Septum pellucidum beschrieben. Ein hypoplastischer 3. Ventrikel kann zur Fusion beider Thalami führen. Auch eine Hypo- bzw. Dysplasie des Corpus callosum wurde beobachtet (Barkovich A. J. Pediatric Neuroimaging. $3^{\text {rd }}$ ed. New York, Raven Press 2000: 346 f; Truwit CL. et al. Am J Neuroradiol 1991; 12: 957).

Differenzialdiagnostisch sollten drei Fehlbildungssyndrome mit Hypo- oder Aplasie des Kleinhirnwurmes abgegrenzt werden:

1. Das Joubert-Syndrom: Hier ist eine typische klinische Symptomatik mit episodischen Hyperventilationen, abnormalen Augenbewegungen, Ataxie und mentaler Retardierung anzutreffen. Die Anomalie der hinteren Schädelgrube umfasst eine Hypoplasie oder Aplasie des Kleinhirnwurmes. Der mittlere Anteil des 4. Ventrikels ist dreieckförmig, der darüberliegende Anteil „batwingshaped“ (Barkovich A. J. Pediatric Neuroimaging. $3^{\text {rd }}$ ed. New York, Raven Press 2000: 346 f).

2. Die Dandy-Walker-Malformation: Sie besteht aus einer partiellen oder kompletten Agenesie des Kleinhirnwurmes, einer zystischen Dilatation des 4. Ventrikels und einer vergrößerten hinteren Schädelgrube mit Kranialverlagerung des Sinus transversus, des Tentorium cerebelli sowie des Torculars (Utsunomiya H. Am J Neuroradiol 1998; 19: 547).

3. Die tectocerebelläre Dysraphie: Sie ist durch eine Hypo- oder Aplasie des Kleinhirnwurms, eine occipitale Cephalocele und eine Dorsaltraktion des Hirnstamms gekennzeichnet. Die hypoplastischen Kleinhirnhemisphären sind um den Hirnstamm rotiert und liegen ventrolateral von diesem (Utsunomiya H. Am J Neuroradiol 1998; 19: 547).

Bei tiefstehenden Kleinhirntonsillen und beim Vorliegen einer lumbalen Myelomeningocele sollte differenzialdiagnostisch auch eine Chiari-Missbildung Typ 2 ausgeschlossen werden. Bei diesem Krankheitsbild liegt jedoch keine Fehlbildung des Kleinhirnwurmes vor (Sener R. N. Comput Med Imaging Graph 2000; 24 (4): 277).
Bei dem von uns untersuchten Kind fielen neben den für eine Rhombencephalosynapsis charakteristischen Kleinhirnfehlbildungen komplexe Anomalien der supratentoriellen Mittellinienstrukturen auf. Es zeigten sich eine Fusion der hypoplastischen Seitenventrikel bei Aplasie des Septum pellucidum und der Fornices sowie eine Aplasie des dorsalen Balkendrittels und eine Asymmetrie der Stammganglien mit partieller Fusion der $\mathrm{Ncl}$. caudati sowie der Thalami. Es bestand Verdacht auf Aquaeduktstenose bei fehlendem Flusssignal. Auch kortikale Entwicklungsstörungen, wie im vorgestellten Fall eine Polymikrogyrie, werden im Rahmen der Rhombencephalosynapsis beobachtet (Barkovich A. J. Pediatric Neuroimaging. $3^{\text {rd }}$ ed. New York Raven Press 2000: 346f).

S. Caffier, T. Kittner, M. Laniado, Dresden 